\title{
Hydration Strategies for Preventing Contrast-Induced Acute Kidney Injury: A Systematic Review and Bayesian Network Meta-Analysis
}

\author{
Qiuping Cai, Ran Jing, Wanfen Zhang, Yushang Tang, Xiaoping Li, and Tongqiang Liu (iD \\ Division of Nephrology, The Affiliated Changzhou NO.2 People's Hospital of Nanjing Medical University, Changzhou 213003, \\ Jiangsu, China \\ Correspondence should be addressed to Tongqiang Liu; liuyf1106@126.com
}

Received 25 May 2019; Accepted 31 December 2019; Published 11 February 2020

Academic Editor: Paul M. Grossman

Copyright ( $) 2020$ Qiuping Cai et al. This is an open access article distributed under the Creative Commons Attribution License, which permits unrestricted use, distribution, and reproduction in any medium, provided the original work is properly cited.

\begin{abstract}
Aims. Many previous studies have examined the effect of different hydration strategies on prevention of contrast-induced acute kidney injury (CI-AKI), but the optimal strategy is unknown. We performed a network meta-analysis (NWM) of these previous studies to identify the optimal strategy. Methods and Results. Web of Science, PubMed, OVID Medline, and Cochrane Library were searched from their inception dates to September 30, 2018. Randomized controlled trials (RCTs) were selected based on strict inclusion criteria, and a Bayesian NWM was performed using WinBUGS V.1.4.3. We finally analyzed 60 eligible RCTs, which examined 21,293 patients and 2232 CI-AKI events. Compared to intravenous $0.9 \%$ sodium chloride (reference), intravenous sodium bicarbonate (OR [95\% CI]: 0.74 [0.57, 0.93]), hemodynamic guided hydration $(0.41[0.18,0.93])$, and RenalGuard guided hydration $(0.32[0.14,0.70])$ significantly reduced the occurrence of CI-AKI. Oral hydration and intravenous $0.9 \%$ sodium chloride were each noninferior to no hydration in preventing CI-AKI. Intravenous $0.9 \%$ sodium chloride, sodium bicarbonate, and hemodynamic guided hydration were each noninferior to oral hydration in preventing CI-AKI. Based on surface under the cumulative ranking curve values, the RenalGuard system was best (0.974) and hemodynamic guided hydration was second best (0.849). Conclusion. There was substantial evidence to support the use of RenalGuard or hemodynamic guided hydration for preventing CI-AKI in high-risk patients, especially those with chronic kidney disease or cardiac dysfunction.
\end{abstract}

\section{Introduction}

Contrast-induced acute kidney injury (CI-AKI), also referred to as contrast-induced nephropathy (CIN), is an iatrogenic complication that can occur following intravascular administration of iodinated contrast medium (CM) prior to radiography. CI-AKI is the third leading cause of hospital-acquired acute renal injury (AKI) [1]. CI-AKI has a low incidence in the general population, but it has a significant incidence in patients with certain risk factors. Moreover, the occurrence of CI-AKI following cardiac catheterization procedures is associated with an in-hospital mortality of $20 \%$, a 1 -year mortality of up to $66 \%$, and an even higher mortality in patients who require dialysis $[2,3]$. However, even if patients with high risk of CI-AKI can be identified a priori, no known pharmaceutical treatment can effectively prevent or treat CI-AKI.

Guidelines recommend intravascular hydration to prevent CI-AKI $[4,5]$, and there are several specific hydration strategies, but researchers have not yet established an optimal strategy [6-9]. Notably, recent randomized controlled trials (RCTs) have led to doubts about the effectiveness of various hydration strategies in prevention of CI-AKI. For example, Nijssen et al. [10] conducted an RCT with 660 highrisk patients and found that no prophylaxis was noninferior or cost-saving relative to intravenous hydration. Weisbord et al. [11] enrolled 5177 high-risk patients and reported no benefit of intravenous sodium bicarbonate relative to normal saline. Another RCT [12] concluded that the benefit of sodium bicarbonate was marginal relative to isotonic 
sodium chloride for preventing CI-AKI among critically ill patients. However, other studies indicated that the RenalGuard System [13-16] and hemodynamic guided hydration [17-19] were safe and effective in preventing CI-AKI. Because of these apparently discrepant results, we conducted a network meta-analysis (NMA) to assess the effects of various hydration strategies on the occurrence of CI-AKI in an effort to identify the optimal strategy for prevention of CI-AKI.

\section{Methods}

2.1. Data Search. This systematic review and meta-analysis were performed according to Cochrane Handbook guidelines [20]. The Web of Science, PubMed, OVID Medline, and Cochrane Library databases were searched using medical subject headings or keywords. Relevant published original studies that were published up to September 30, 2018, were examined. The search syntax was as follows: "contrast-induced acute kidney injury OR contrast-induced nephropathy OR CIN OR CI-AKI OR contrast acute renal failure OR contrast nephropathy" AND "hydration OR fluid administration $\mathrm{OR}$ volume expansion $\mathrm{OR}$ intravenous sodium bicarbonate OR saline infusion.”

2.2. Study Selection. An initial eligibility screen of all citations was conducted, and only studies that examined CI-AKI and hydration were selected for further full-text review. All included studies were RCTs; experimental studies were excluded. In addition, all included studies reported the prevention of CI-AKI after intravascular administration of CM; used clinical protocols that were hydration strategies, not pharmaceutical prevention strategies; had clear definitions of CI-AKI; and provided data on the outcome of interest (occurrence of CI-AKI within 2 days to 1 week after procedures).

2.3. Data Extraction and Quality Assessment. Two authors (C. Q. P. and J. R.) independently reviewed each article for eligibility. Any disagreement was resolved by discussion among the authors or involvement of a third author. Data extraction included the year of publication, sample size, patient characteristics, risk factors associated with CI-AKI (old age, diabetes mellitus, renal impairment, heart failure), and type and dosage of contrast medium. The primary endpoint was the occurrence of CI-AKI within 2 days to 1 week after intravascular administration of CM. Two investigators independently evaluated the quality of each study using the Jadad scale, which ranges from 0 (worst) to 5 (best) [21].

2.4. Statistical Analyses. The advantages of Bayesian NMA over traditional meta-analysis are its greater flexibility, its provision of more naturally interpretable results, and its ability to rank treatments by comparative effectiveness [22]. The occurrence of CI-AKI as a dichotomous outcome variable was expressed as an odds ratio (OR) and 95\% confidence interval (CI). All $P$ values were 2 -sided, and a $P$ value below 0.05 was considered significant. All analyses were conducted using the Bayesian Markov chain Monte Carlo method in WinBUGS V.1.4.3 (MRC Biostatistics Unit, Cambridge, United Kingdom) using the Microsoft Excelbased macro NetMetaXL V.1.6.1 (Canadian Agency for Drugs and Technologies in Health, Ottawa, Canada) [23]. A convergence test for each analysis was conducted by checking whether the Monte Carlo error was less than 5\% of the SD of the effect estimates or the variance between the studies. Convergence was achieved for all analyses using 1000 "burn in" runs and 1000 model runs. NetMetaXL was also used to generate a forest plot, league table, and "rankogram" with surface under the cumulative ranking curve (SUCRA), which ranges from 0 (worst) to $100 \%$ (best). Inconsistency was assessed by comparing the residual deviance and deviance information criterion statistics in fitted consistency and inconsistency models.

\section{Results}

3.1. Literature Search. We initially identified 3620 publications, assessed 703 RCTs for eligibility by review of the full texts, and ultimately included 60 RCTs which met the eligibility criteria (Figure 1). These studies examined 21,293 patients (median: 222, interquartile range [IQR]: 120, 350) and 2232 CI-AKI events. All included RCTs were full-length journal articles. Agreement between the two reviewers at the full-text review stage was excellent (Cohen's $\kappa=0.85$ ).

3.2. Characteristics of Studies and Participants. Table 1 shows the characteristics of the included studies. The publication date ranged from 2002 to 2018 , and about $50 \%$ of the studies were published after 2013. The proportion of male patients ranged from $25.0 \%$ to $98.1 \%$ (median [IQR]: 65.7 [56.9, $74.8]$ ), and the mean age ranged from 56.2 to 82.9 years $(67.8$ $[63.1,72.5])$. Thirty-one studies enrolled 12,519 patients who had high risk of CI-AKI. The baseline serum creatinine (SCr) level ranged from 61.4 to $236.4 \mu \mathrm{mol} / \mathrm{L}(117.1$ [89.5, 136.9]), and the baseline estimated glomerular filtration rate (eGFR) ranged from 32 to $93.1 \mathrm{~mL} / \mathrm{min} / 1.73 \mathrm{~m}^{2}$ (49.2 [44.1, 74.2]). Twenty-three studies provided the values of left ventricular ejection fraction (LVEF); the mean LVEF ranged from $25 \%$ to $57.8 \%(49.0[42.8,54.5])$. The percentage of diabetes mellitus (DM) patients ranged from $8 \%$ to $100 \%$, and the percentage with heart failure (HF) ranged from $0.6 \%$ to $45.8 \%$. A total of 8176 patients from 32 studies received intravenous low-osmolar nonionic CM, 9993 patients from 17 studies received iso-osmolar nonionic CM, and 317 patients from 2 studies received low-osmolar ionic CM. The mean Jadad score of the 60 RCTs was $3.2(3[2,4])$, indicating the overall study quality was good.

3.3. Network Meta-Analysis. Figure 2 shows all the comparisons in the NMA. Thirty-seven studies (13,365 participants) compared the efficacy of intravenous sodium bicarbonate and $0.9 \%$ sodium chloride. The other hydration strategies were nonhydration (8 studies, 1396 patients), oral hydration (6 studies, 355 patients), intravenous half isoosmolar saline (3 studies, 968 patients), intravenous 


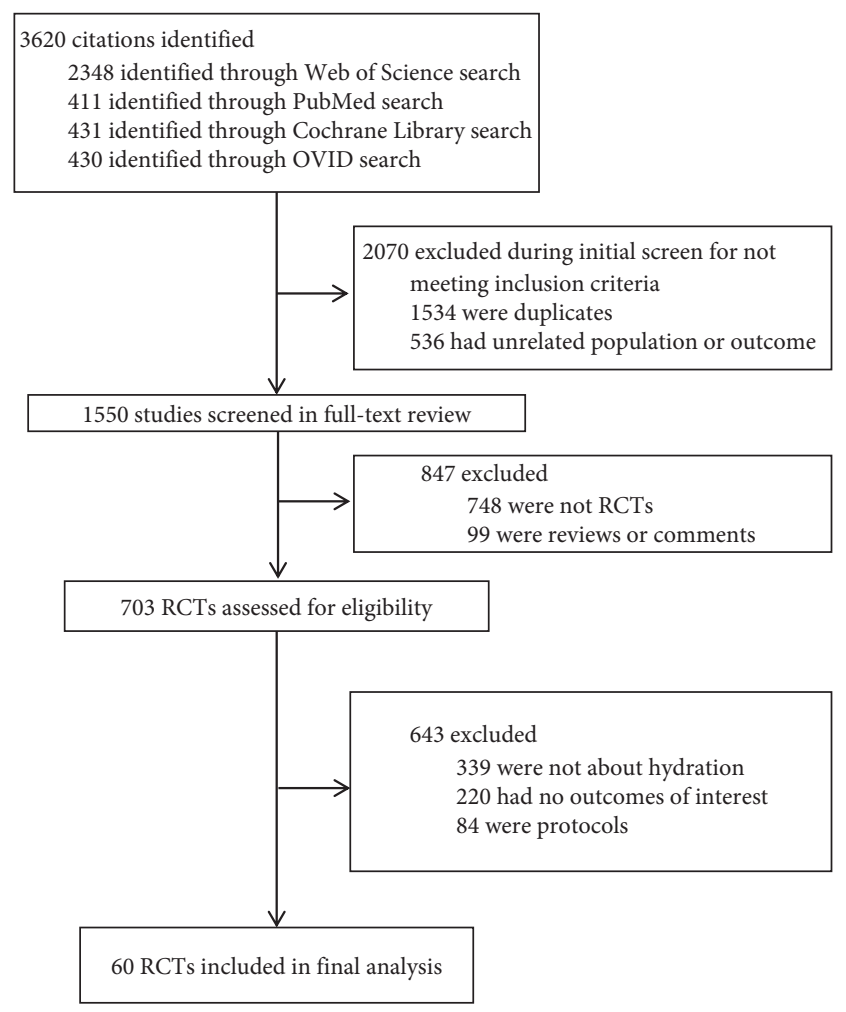

Figure 1: Identification and selection of studies for Bayesian network meta-analysis.

hydration, mainly normal saline + diuresis (2 studies $[26,31], 501$ patients), hemodynamic guided hydration (3 studies, 458 patients), and RenalGuard system guided hydration (4 studies, 348 patients).

We compared the ORs of the different hydration strategies using a forest plot (Figure 3) and analyzed the results of the random effects consistency NMA using a league table, which shows all pairwise comparisons (Figure 4). Taken together, these results indicate that, relative to typical intravenous $0.9 \%$ sodium chloride hydration (reference), the occurrence of CI-AKI was significantly reduced by intravenous sodium bicarbonate (OR [95\% CI]: 0.74 [0.57, 0.93]), hemodynamic guided hydration $(0.41[0.18,0.93])$, and RenalGuard system guided hydration $(0.32[0.14,0.70])$. Oral hydration $(0.72[0.28,1.82])$ and intravenous $0.9 \%$ sodium chloride $(0.64[0.39,1.08])$ were each noninferior to no hydration for prevention of CI-AKI. Relative to oral hydration (reference), intravenous $0.9 \%$ sodium chloride or sodium bicarbonate and hemodynamic guided hydration were each noninferior in prevention of CI-AKI, but RenalGuard guided hydration was superior $(0.21$ [0.07, $0.63]$ ). Intravenous hydration plus diuresis also did not decrease the risk of CI-AKI relative to oral hydration and no hydration.

A rankogram and SUCRA values indicated the RenalGuard system was best (SUCRA $=0.974)$ followed by hemodynamic guided hydration (SUCRAs $=0.849$; Figure 5). Intravenous sodium bicarbonate had a SUCRA of 0.667 . The SUCRAs for intravenous $0.9 \%$ sodium chloride, intravenous hydration plus diuresis, oral and no hydration, and the other treatments ranged from 0.197 to 0.441 , and their rankings were similar. Hydration using half iso-osmolar saline alone was the least effective treatment.

3.4. Inconsistency Analysis. We performed network inconsistency assessment for the fixed effect model for the 60 studies (Figure 6). The resulting plot demonstrated that nearly all the studies were near the line of equality and that the results were therefore consistent. However, there was some evidence of inconsistency in 3 noninferiority studies [10, 31]. In particular, Martin-Moreno et al. [31] and Nijssen et al. [10] found that intravenous sodium bicarbonate and $0.9 \%$ sodium chloride were noninferior to oral hydration.

\section{Discussion}

To our knowledge, this is the first NMA to compare different hydration strategies for prevention of CI-AKI. We included 60 RCTs which examined 21,293 participants and 2232 CIAKI events. Our comparison of 8 hydration strategies for preventing CI-AKI confirmed that, relative to intravenous $0.9 \%$ sodium chloride hydration, three treatments during CM administration significantly reduced the risk for CIAKI: the RenalGuard system, hemodynamic guided hydration, and intravenous sodium bicarbonate. Relative to no hydration, oral hydration and intravenous $0.9 \%$ sodium chloride were each noninferior in prevention of CI-AKI. Relative to oral hydration, intravenous $0.9 \%$ sodium chloride and sodium bicarbonate were each noninferior in prevention of CI-AKI. Thus, we ranked the RenalGuard system as the best strategy and hemodynamic guided hydration as the second best.

Guidelines for the prevention of CI-AKI in high-risk patients routinely recommend hydration protocols before contrast exposure as an established preventive measure $[77,78]$. A recent large RCT [10] led us to reanalyze the efficacy of hydration for prevention of CI-AKI. In particular, the AMAstricht Contrast-Induced Nephropathy Guideline (AMACING) study [10] enrolled 660 patients with high risk of CI-AKI and concluded that, relative to intravenous hydration, no prophylaxis was less expensive and noninferior in prevention of CI-AKI. In our meta-analysis, five studies compared the effectiveness of intravenous $0.9 \%$ sodium chloride and three studies compared bicarbonate with nonhydration, leading to our conclusion that, relative to no hydration (reference), oral hydration or hydration with intravenous $0.9 \%$ sodium chloride was noninferior in prevention of CI-AKI. These results were unsurprising, because simple oral or intravenous hydration can lead to complications, such as heart failure, pulmonary edema, and electrolyte disorders. Thus, the safety window of hydration is relatively narrow for patients undergoing percutaneous coronary intervention (PCI), and other more effective or precise hydration strategies may be needed to decrease the incidence of CI-AKI.

Most meta-analyses before 2016 [79-83] confirmed that intravenous sodium bicarbonate was more effective than sodium chloride in preventing CI-AKI. However, two recent 


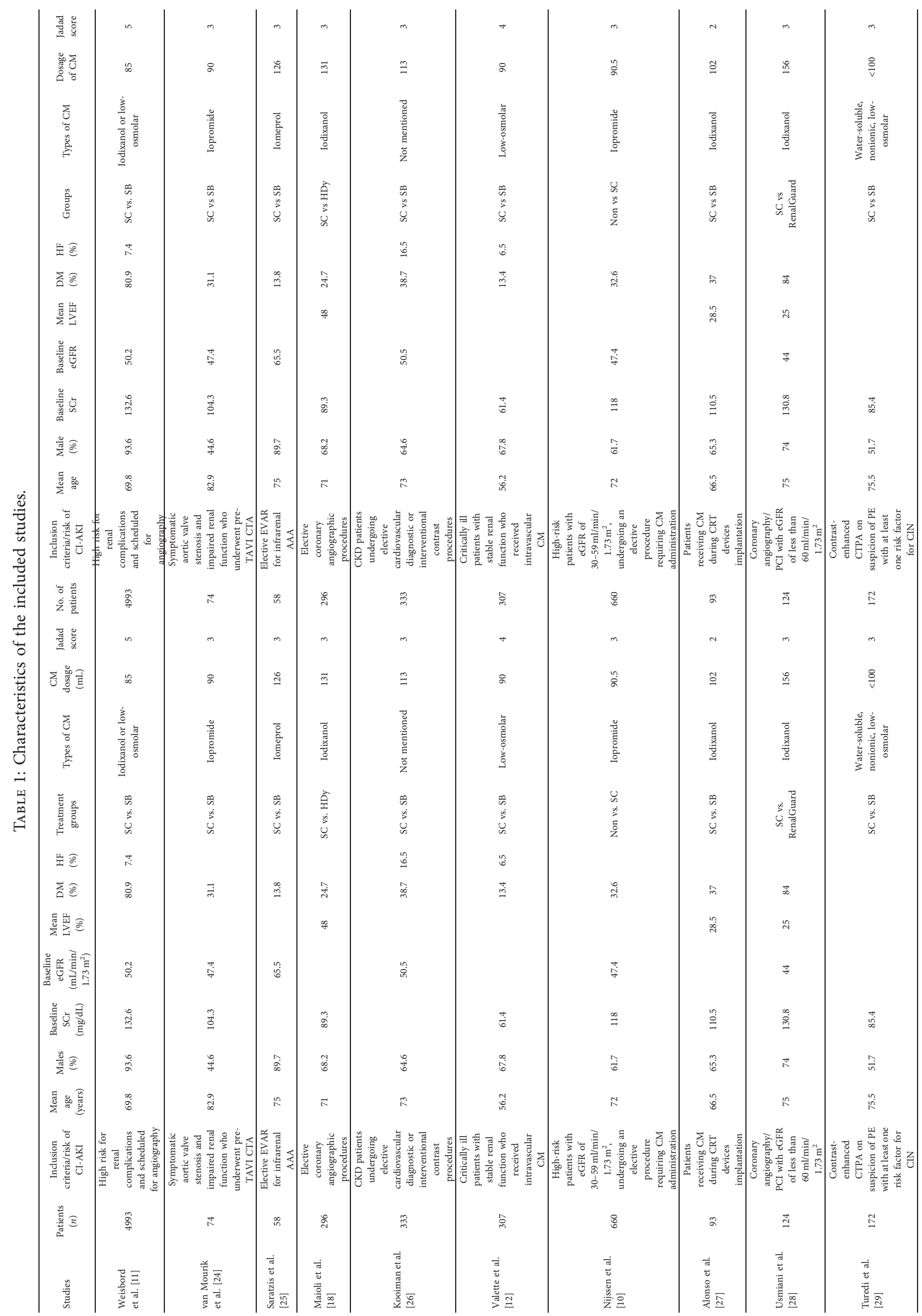




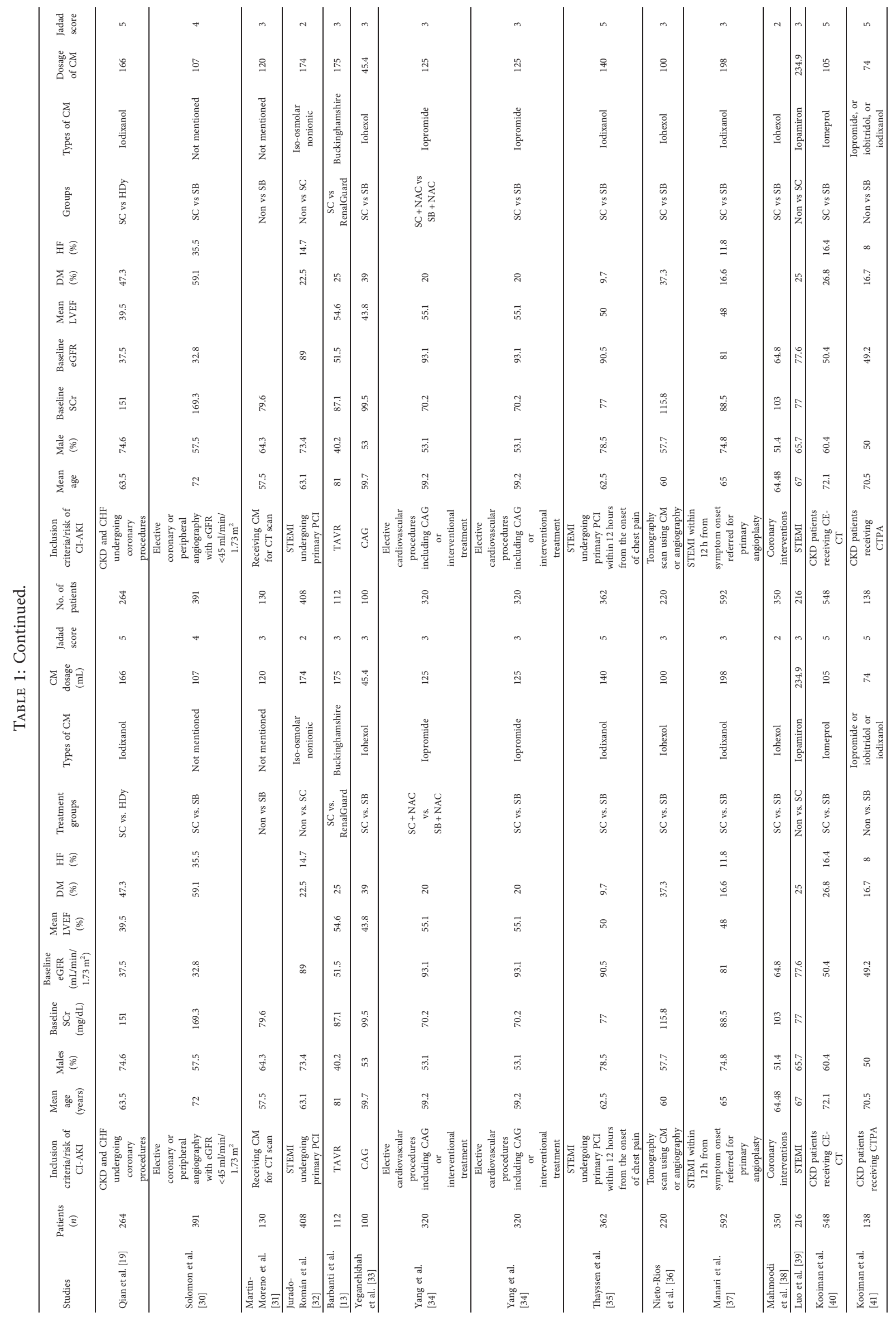




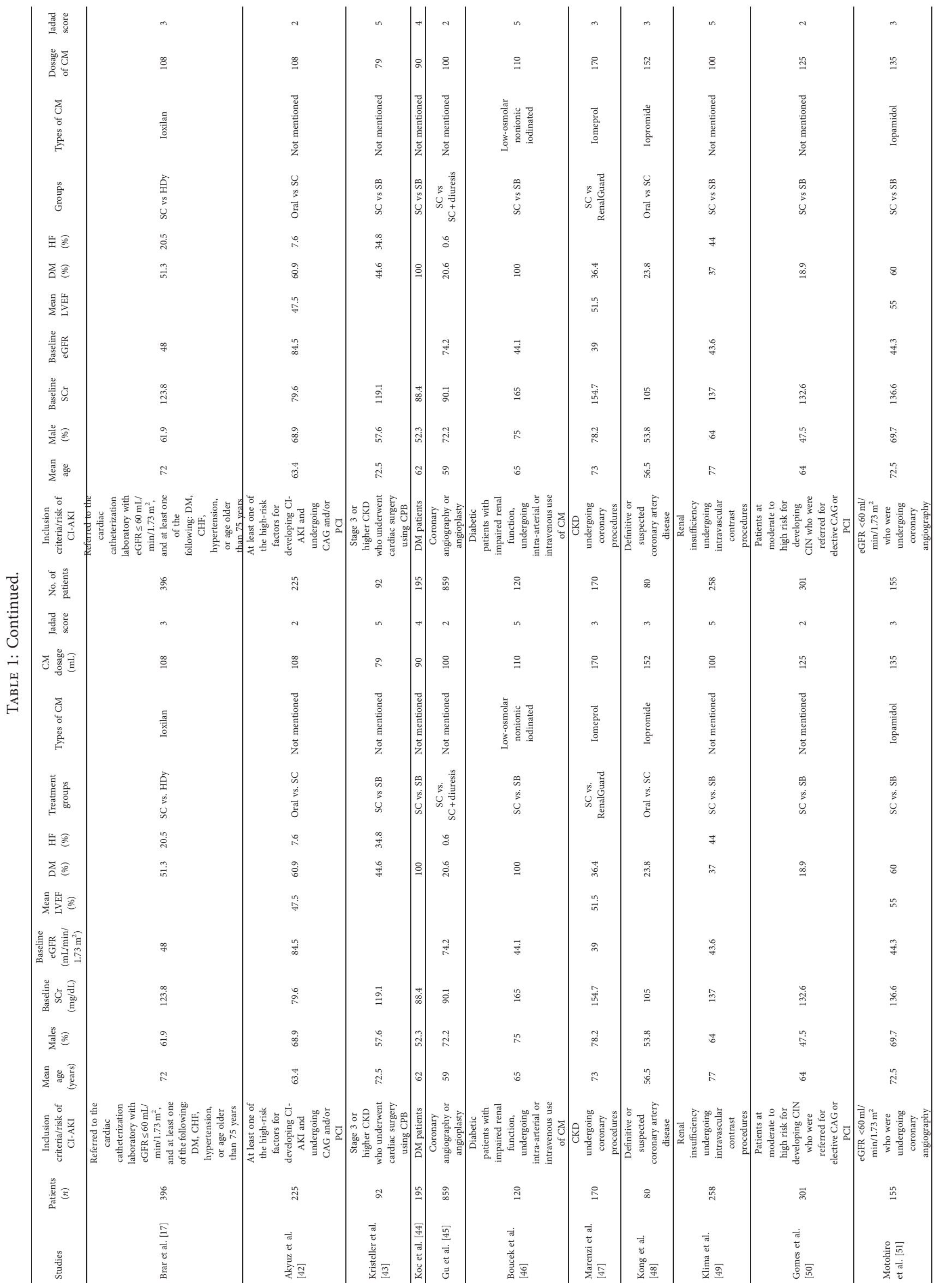




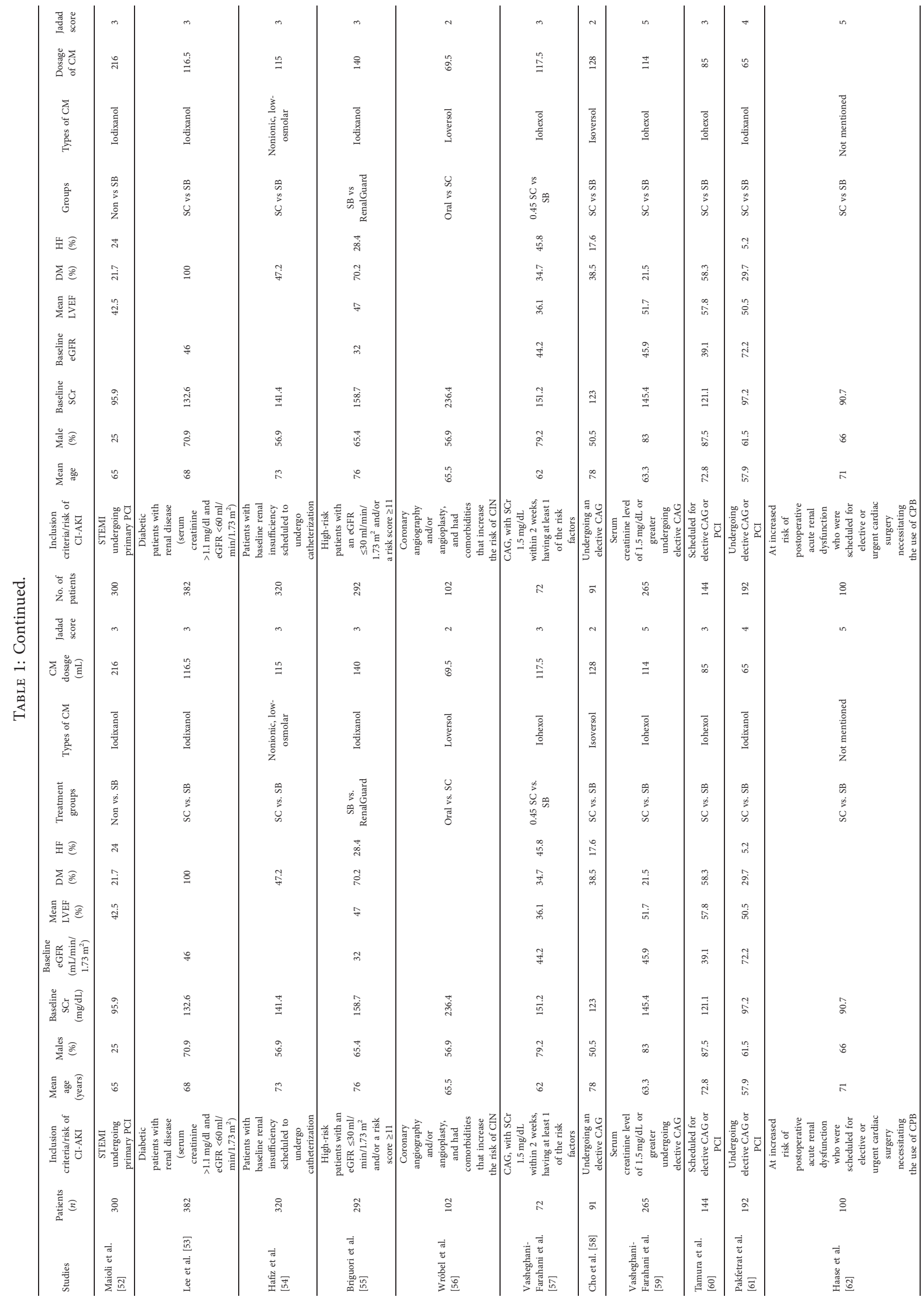




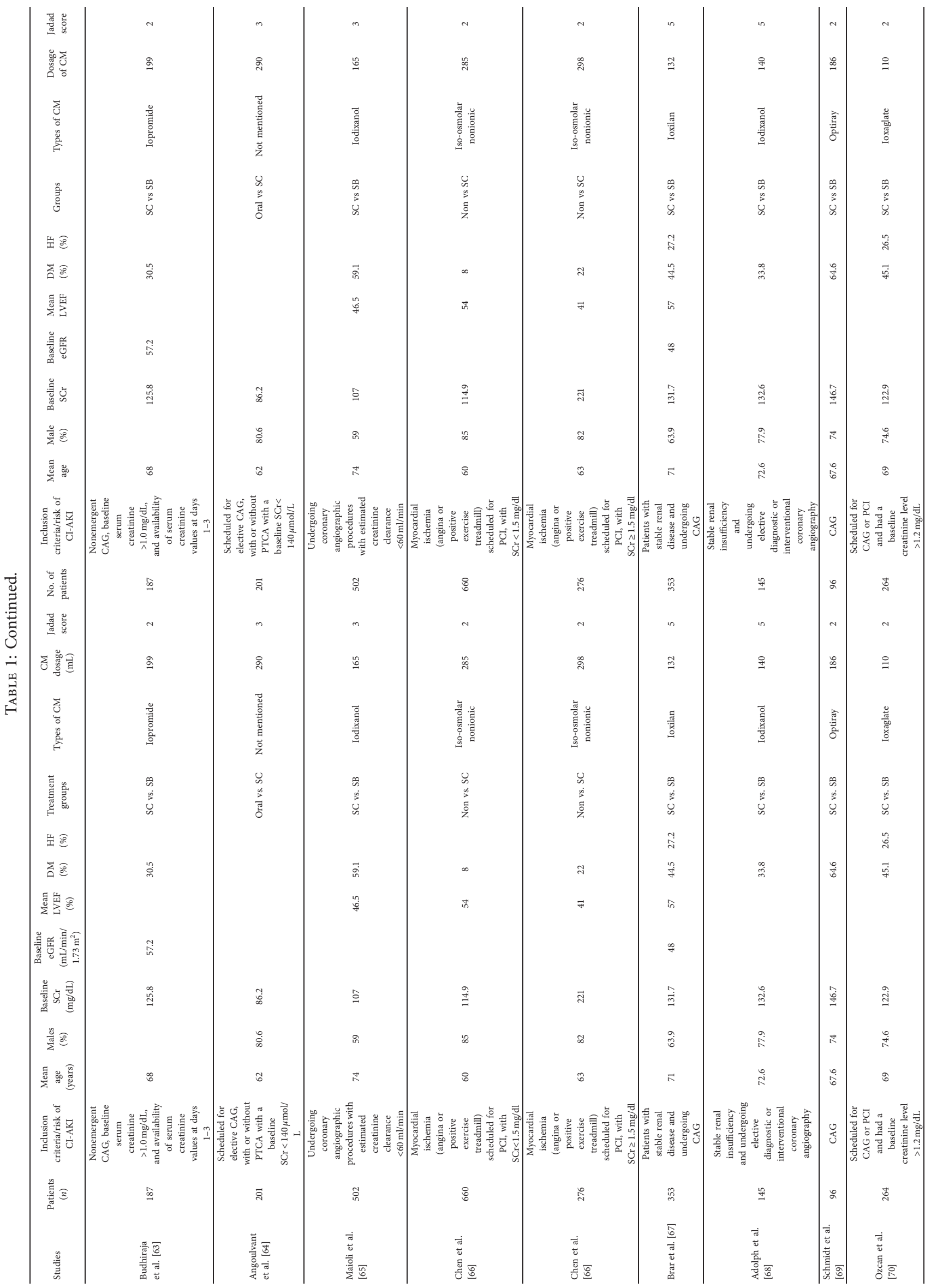




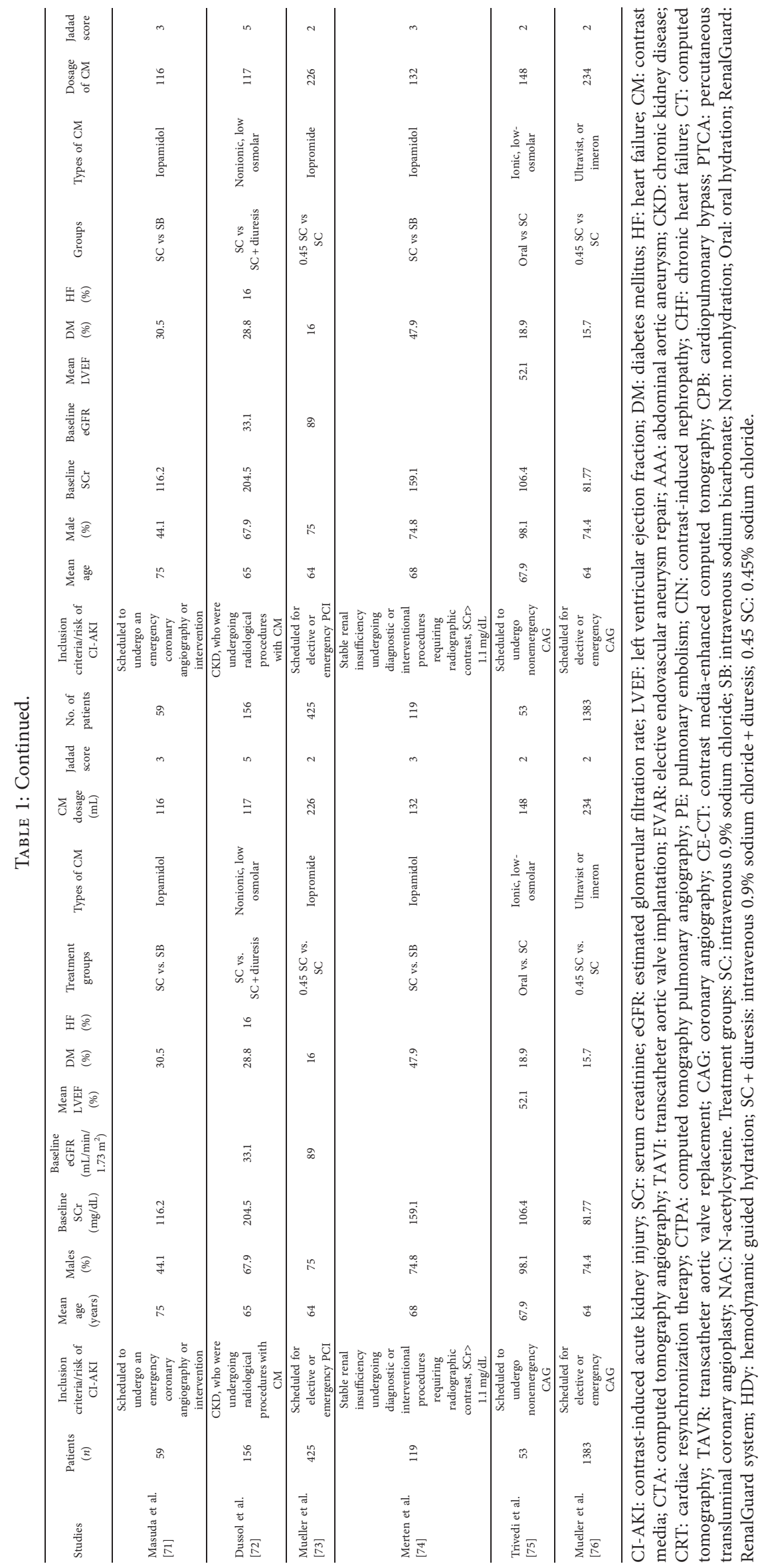




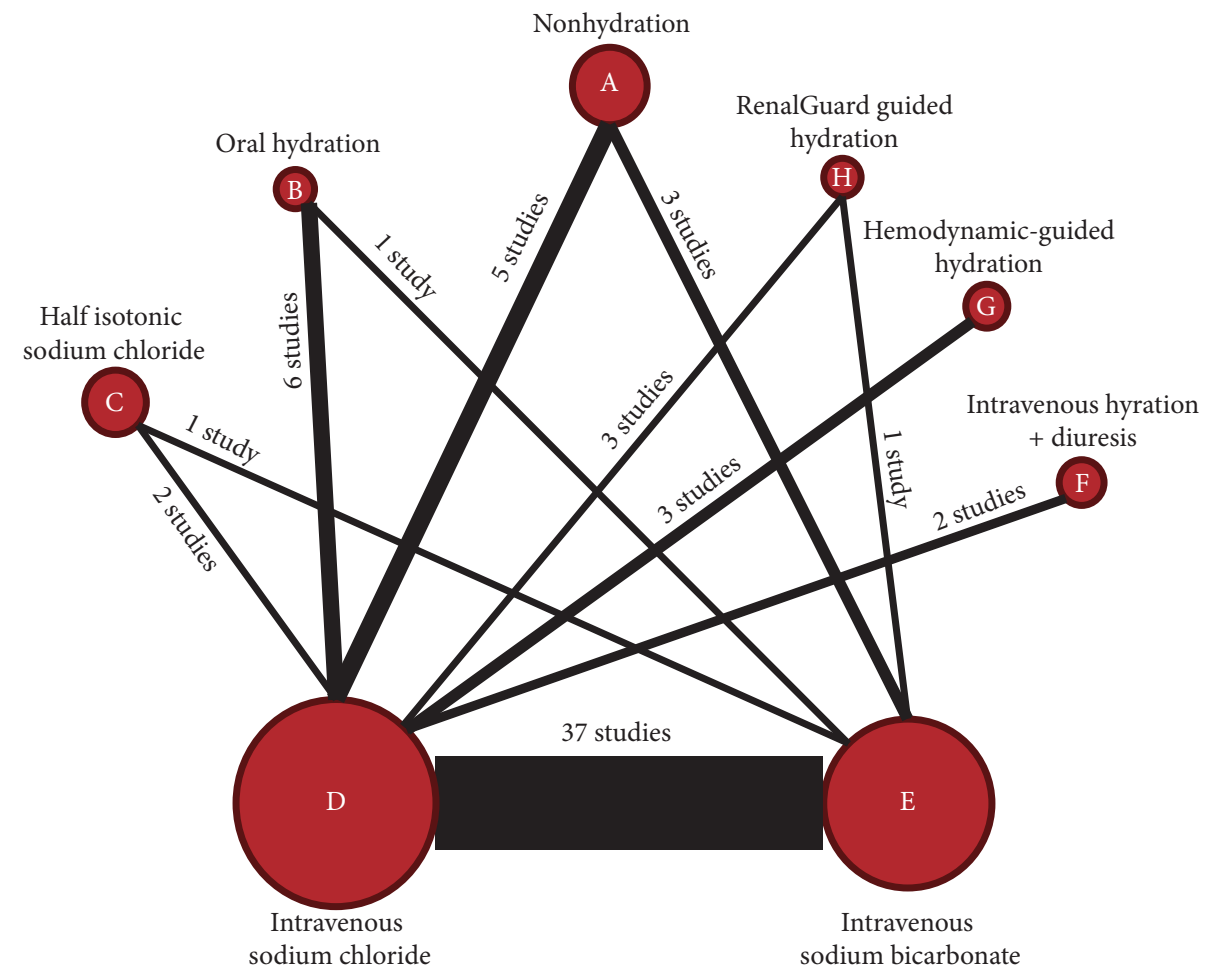

Figure 2: Network diagram of eight hydration strategies used to prevent contrast-induced acute kidney injury in the 60 included studies. Circles represent hydration strategies and lines represent direct comparisons. Circle size indicates the number of participants who received each treatment, and line thickness indicates the number of studies in each comparison.

influential studies concluded that intravenous sodium bicarbonate provided no benefit over intravenous sodium chloride in high-risk patients [11] and critically ill patients [12]. Our NMA included 37 studies that compared intravenous sodium chloride with sodium bicarbonate, and our results also indicated that intravenous sodium bicarbonate led to a reduced risk for CI-AKI, although the effect size was small (OR [95\% CI]: 0.74 [0.57, 0.93]). Alkalization with bicarbonate perfusion could theoretically reduce the formation of reactive oxygen species by decreasing the production of hydroxyl radicals due to inhibition of the Haber-Weiss and Fenton reactions [84]. However, the HYDRAREA study [12] assessed 307 critically ill patients with stable renal function and found that hydration with bicarbonate provided no benefit relative to hydration with isotonic sodium chloride. These researchers also noted that bicarbonate provided a greater benefit in the smaller studies, suggesting publication bias. Recently, Weisbord et al. [11] enrolled 5177 patients with high risk for renal complications and found that administration of sodium bicarbonate did not reduce the occurrence of CI-AKI. This result supports the interpretation that sodium bicarbonate is not more effective than sodium chloride in preventing CI-AKI or longer-term adverse outcomes after angiography. However, there was high heterogeneity among our 60 studies regarding concurrent medications, comorbidities (CHF, DM), types of CM, periprocedural hydration protocols, concentrations and dosages of sodium bicarbonate, and radiographic procedures [12]. Thus, we do not recommend alkalization with intravenous sodium bicarbonate as a single strategy, and a more effective hydration strategy is needed to prevent CI-AKI.

Several recent RCTs of high risk patients [13, 28, 47, 55] showed that furosemide-induced high-volume forced diuresis with matched hydration using the RenalGuard system effectively prevented CI-AKI. RenalGuard is a closed-loop fluid-management system, in which each volume of urine that enters the collection bag leads to the infusion of an equal volume of saline into the patient. Two meta-analyses $[14,16]$ of RCTs concluded that the RenalGuard system significantly reduced the risk of CI-AKI and the need for renal replacement therapy in high-risk patients undergoing coronary angiography. Our rankogram analysis indicated that the RenalGuard system of guided hydration had the highest rank, with a SUCRA of 0.974 . However, we did not assess the effectiveness of intravenous hydration plus diuresis without a guided system, and the rankogram indicated that hemodynamic guided hydration was the second best method, with a SUCRA of 0.849. Brar et al. [17] used left ventricular end-diastolic pressure to guide fluid administration and demonstrated that this method was safe and effective in prevention of CI-AKI among patients undergoing cardiac catheterization. Another study [19] demonstrated that central venous pressure-guided fluid administration safely and effectively reduced the risk of CIAKI in patients with CKD and CHF. Maioli et al. [18] assessed body fluid level using bioimpedance vector analysis (BIVA), which allows adjustment of intravascular 


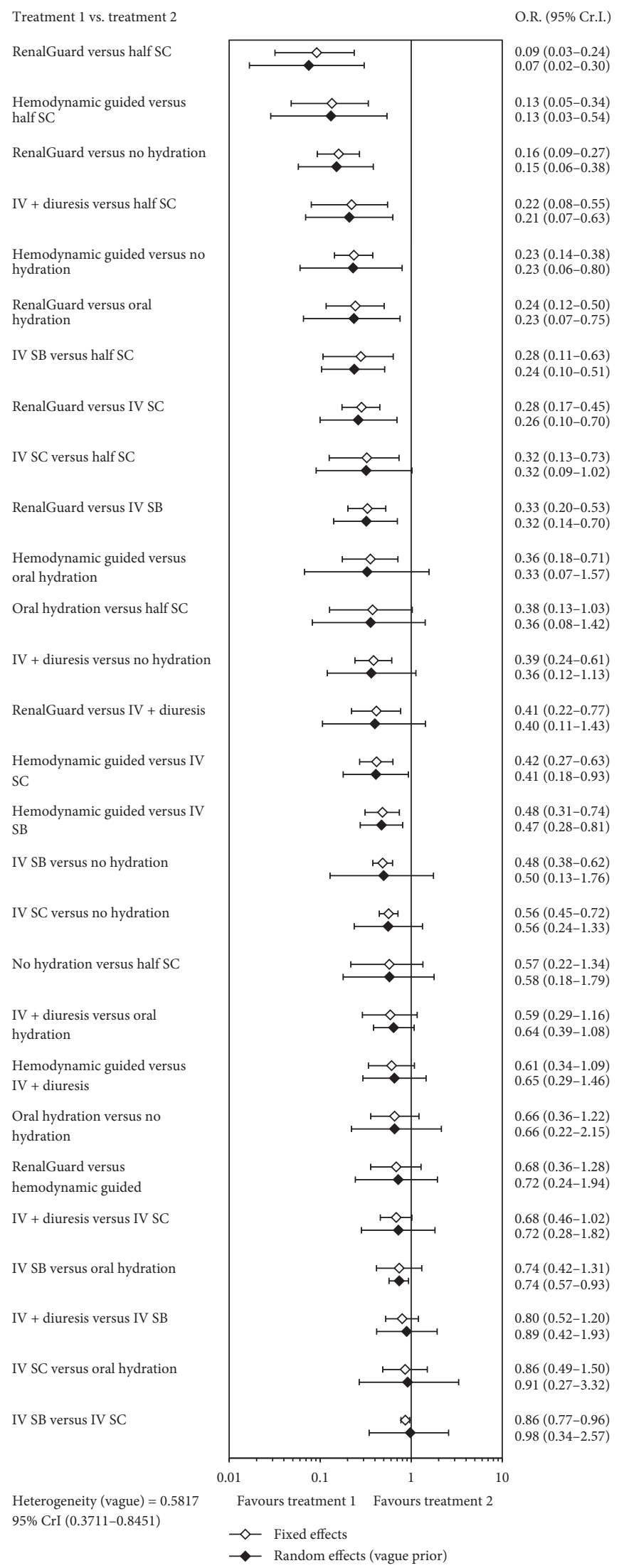

FIGURE 3: Forest plot showing the effect of different hydration strategies. Summary estimates from the pooled studies with $95 \%$ confidence intervals are indicated for fixed effects (open diamonds) and random effects (filled diamonds) models. 
OR $<1$ means the treatment in top left is better

\begin{tabular}{|c|c|c|c|c|c|c|c|}
\hline RenalGuard & & & & & & & \\
\hline $\begin{array}{c}0.58 \\
(0.18-1.79)\end{array}$ & $\begin{array}{c}\text { Hemodynamic } \\
\text { guided }\end{array}$ & & & & & & \\
\hline $\begin{array}{c}0.32 \\
(0.14-0.70)\end{array}$ & $\begin{array}{c}0.56 \\
(0.24-1.33)\end{array}$ & IV SB & & & & & \\
\hline $\begin{array}{c}0.24 \\
(0.10-0.51)\end{array}$ & $\begin{array}{c}0.41 \\
(0.18-0.93)\end{array}$ & $\begin{array}{c}0.74 \\
(0.57-0.93)\end{array}$ & IV SC & & & & \\
\hline $\begin{array}{c}0.23 \\
(0.06-0.80)\end{array}$ & $\begin{array}{c}0.40 \\
(0.11-1.43)\end{array}$ & $\begin{array}{c}0.72 \\
(0.24-1.94)\end{array}$ & $\begin{array}{c}0.98 \\
(0.34-2.57)\end{array}$ & $\mathrm{IV}+$ diuresis & & & \\
\hline $\begin{array}{c}0.21 \\
(0.07-0.63)\end{array}$ & $\begin{array}{c}0.36 \\
(0.12-1.13)\end{array}$ & $\begin{array}{c}0.65 \\
(0.29-1.46)\end{array}$ & $\begin{array}{c}0.89 \\
(0.42-1.93)\end{array}$ & $\begin{array}{c}0.91 \\
(0.27-3.32)\end{array}$ & Oral hydration & & \\
\hline $\begin{array}{c}0.15 \\
(0.06-0.38)\end{array}$ & $\begin{array}{c}0.26 \\
(0.10-0.70)\end{array}$ & $\begin{array}{c}0.47 \\
(0.28-0.81)\end{array}$ & $\begin{array}{c}0.64 \\
(0.39-1.08)\end{array}$ & $\begin{array}{c}0.66 \\
(0.22-2.15)\end{array}$ & $\begin{array}{c}0.72 \\
(0.28-1.82)\end{array}$ & No hydration & \\
\hline $\begin{array}{c}0.07 \\
(0.02-0.30)\end{array}$ & $\begin{array}{c}0.13 \\
(0.03-0.54)\end{array}$ & $\begin{array}{c}0.23 \\
(0.07-0.75)\end{array}$ & $\begin{array}{c}0.32 \\
(0.09-1.02)\end{array}$ & $\begin{array}{c}0.33 \\
(0.07-1.57)\end{array}$ & $\begin{array}{c}0.36 \\
(0.08-1.42)\end{array}$ & $\begin{array}{c}0.50 \\
(0.13-1.76)\end{array}$ & Half SC \\
\hline
\end{tabular}

Figure 4: League table, showing all pairwise comparisons of studies.

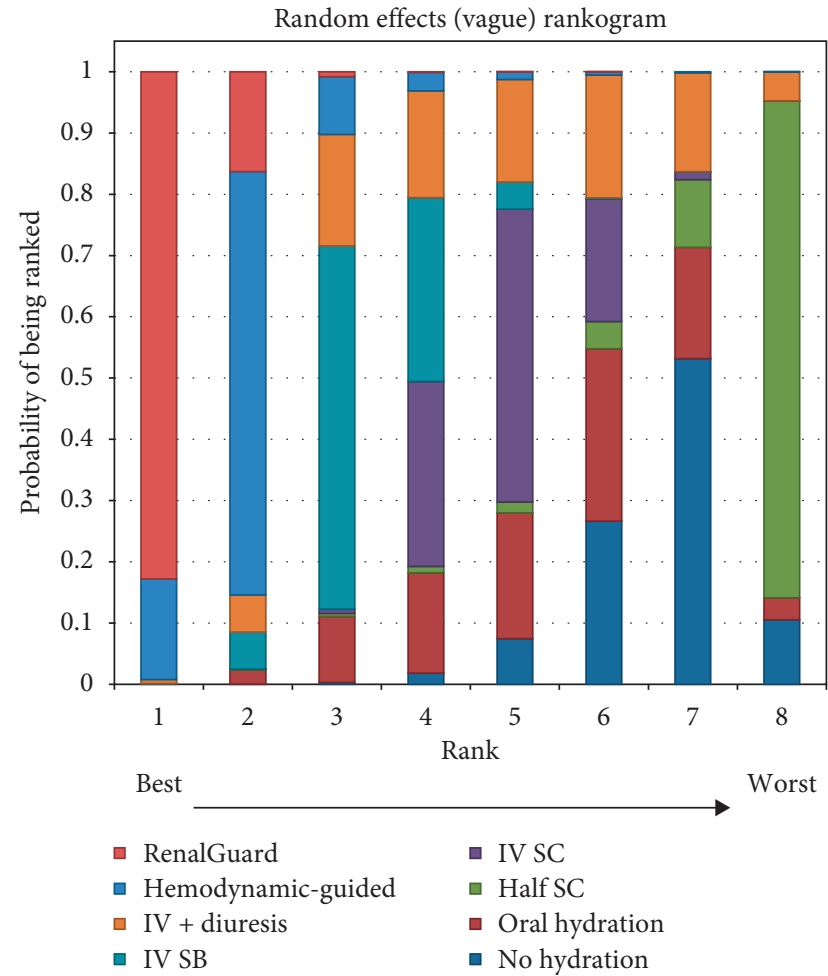

FIGURE 5: Rankogram of the effect of different hydration strategies in reducing the risk of contrast-induced acute kidney injury.

volume expansion, and this led to a lower incidence of CIAKI after angiographic procedures. Therefore, our results indicate that the RenalGuard system and hemodynamic guided hydration are best for patients with high-risk for CIAKI, especially those with CKD and cardiac dysfunction.

\section{Limitations}

It is essential to note several limitations of our study. Firstly, the hydration protocol should have a substantial

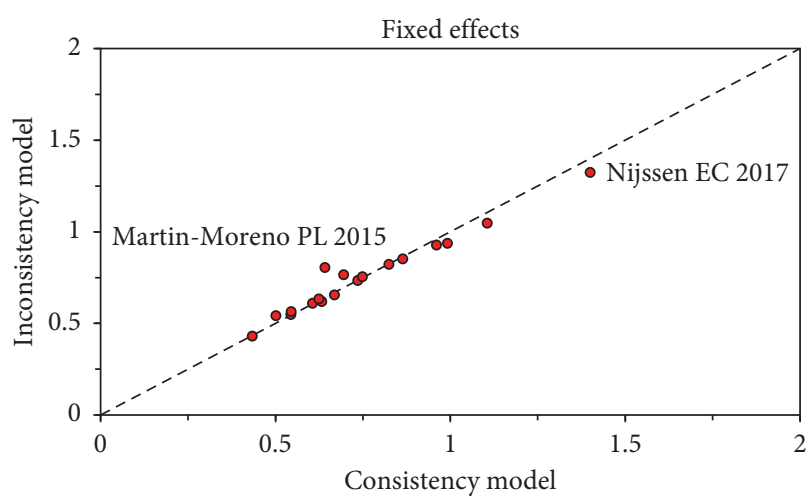

FIgURE 6: Inconsistency plot of enrolled studies, showing the posterior mean deviance of each study from the consistency model (horizontal axis) and the inconsistency model (vertical axis).

influence on CI-AKI, but because of the high heterogeneity of specific protocols used in the included studies, we could not analyze distinct protocols, such as the effect of different concentrations of sodium bicarbonate, and the effect of hydration duration. Secondly, several confounding factors that we did consider may have impacted the effects of hydration, including dosage and types of $\mathrm{CM}$, risk status of patients for CI-AKI, and other factors. Finally, it may be inappropriate to define hemodynamic guided hydration based on the use of different indexes, such as left ventricular end-diastolic pressure, central venous pressure, and bioimpedance.

\section{Conclusion}

This Bayesian NMA provided substantial evidence to support the use of RenalGuard or hemodynamic guided hydration to prevent CI-AKI in high-risk patients, especially those with CKD or cardiac dysfunction. 


\section{Abbreviations}

CI- Contrast-induced acute kidney injury

AKI:

SCr: $\quad$ Serum creatinine

eGFR: Estimated glomerular filtration rate

LVEF: Left ventricular ejection fraction

DM: Diabetes mellitus

HF: Heart failure

CM: Contrast medium

CTA: Computed tomography angiography

TAVI: Transcatheter aortic valve implantation

EVAR: Elective endovascular aneurysm repair

AAA: Abdominal aortic aneurysm

CKD: Chronic kidney disease

CRT: Cardiac resynchronization therapy

CTPA: Computed tomography pulmonary angiography

PE: $\quad$ Pulmonary embolism

CIN: Contrast-induced nephropathy

CHF: Chronic heart failure

CT: $\quad$ Computed tomography

TAVR: Transcatheter aortic valve replacement

CAG: Coronary angiography

CE-CT: Contrast media-enhanced computed tomography

CPB: Cardiopulmonary bypass

PTCA: Percutaneous transluminal coronary angioplasty.

\section{Conflicts of Interest}

All authors have no conflicts of interest and no relationships with industry.

\section{Authors' Contributions}

Qiuping Cai and Ran Jing contributed equally to this paper. C. Q. P. and J. R. carried out meta-analysis, participated in data extraction, and drafted manuscript. Z. W. F. and T. Y. S. carried out quality assessment. L. X. P. participated in study design and performed statistical analysis. L. T. Q. conceived the study and participated in its design and coordination and helped to draft the manuscript. All authors read and approved the final manuscript.

\section{Acknowledgments}

This work was supported by grants from the National Natural Science Foundation of China (81670627), Changzhou Health and Family Planning Commission Major Sci and Tech Projects of China (ZD201501), Changzhou Health and Family Planning Commission Youth Foundation of China (QN201814), and Changzhou Sci and Tech Program of China (CJ20159042).

\section{References}

[1] T. G. Gleeson and S. Bulugahapitiya, "Contrast-induced nephropathy," American Journal of Roentgenology, vol. 183, no. 6, pp. 1673-1689, 2004.

[2] C. S. Rihal, S. C. Textor, D. E. Grill et al., "Incidence and prognostic importance of acute renal failure after percutaneous coronary intervention," Circulation, vol. 105, no. 19, pp. 2259-2264, 2002.

[3] P. A. McCullough, R. Wolyn, L. L. Rocher, R. N. Levin, and W. W. O'Neill, "Acute renal failure after coronary intervention: incidence, risk factors, and relationship to mortality," The American Journal of Medicine, vol. 103, no. 5, pp. 368375, 1997.

[4] H. S. Thomsen and S. K. Morcos, "Contrast media and the kidney: European Society of Urogenital Radiology (ESUR) guidelines," The British Journal of Radiology, vol. 76, no. 908, pp. 513-518, 2003.

[5] J. A. Kellum, N. Lameire, P. Aspelin et al., "Kidney disease: improving global outcomes (KDIGO) acute kidney injury work group. KDIGO clinical practice guideline for acute kidney injury," Kidney International Supplements, vol. 2, pp. 1-138, 2012.

[6] W. Zhang, J. Zhang, B. Yang et al., "Effectiveness of oral hydration in preventing contrast-induced acute kidney injury in patients undergoing coronary angiography or intervention: a pairwise and network meta-analysis," Coronary Artery Disease, vol. 29, no. 4, pp. 286-293, 2018.

[7] Y. Jiang, M. Chen, Y. Zhang et al., "Meta-analysis of prophylactic hydration versus no hydration on contrast-induced acute kidney injury," Coronary Artery Disease, vol. 28, no. 8, pp. 649-657, 2017.

[8] S. K. Agarwal, S. Mohareb, A. Patel et al., "Systematic oral hydration with water is similar to parenteral hydration for prevention of contrast-induced nephropathy: an updated meta-analysis of randomised clinical data," Open Heart, vol. 2, Article ID e000317, 2015.

[9] S. Zoungas, T. Ninomiya, R. Huxley et al., "Systematic review: sodium bicarbonate treatment regimens for the prevention of contrast-induced nephropathy," Annals of Internal Medicine, vol. 151, no. 9, pp. 631-638, 2009.

[10] E. C. Nijssen, R. J. Rennenberg, P. J. Nelemans et al., "Prophylactic hydration to protect renal function from intravascular iodinated contrast material in patients at high risk of contrast-induced nephropathy (AMACING): a prospective, randomised, phase 3, controlled, open-label, non-inferiority trial," The Lancet, vol. 389, no. 10076, pp. 1312-1322, 2017.

[11] S. D. Weisbord, M. Gallagher, H. Jneid et al., "Outcomes after angiography with sodium bicarbonate and acetylcysteine," New England Journal of Medicine, vol. 378, no. 7, pp. 603-614, 2018.

[12] X. Valette, I. Desmeulles, B. Savary et al., "Sodium bicarbonate versus sodium chloride for preventing contrast-associated acute kidney injury in critically ill patients: a randomized controlled trial," Critical Care Medicine, vol. 45, no. 4, pp. 637-644, 2017.

[13] M. Barbanti, S. Gulino, P. Capranzano et al., "Acute kidney injury with the RenalGuard system in patients undergoing transcatheter aortic valve replacement: the PROTECT-TAVI trial (PROphylactic effecT of furosEmide-induCed diuresis with matched isotonic intravenous hydraTion in transcatheter aortic valve implantation)," JACC: Cardiovascular Interventions, vol. 8, no. 12, pp. 1595-1604, 2015.

[14] A. Putzu, M. Boscolo Berto, A. Belletti et al., "Prevention of contrast-induced acute kidney injury by furosemide with matched hydration in patients undergoing interventional procedures: a systematic review and meta-analysis of randomized trials," JACC: Cardiovascular Interventions, vol. 10, no. 4, pp. 355-363, 2017.

[15] G. Visconti, A. Focaccio, M. Donahue et al., "RenalGuard system for the prevention of acute kidney injury in patients 
undergoing transcatheter aortic valve implantation," EuroIntervention, vol. 11, no. 14, pp. e1658-e1661, 2016.

[16] R. Shah, S. J. Wood, S. A. Khan, A. Chaudhry, M. Rehan Khan, and M. S. Morsy, "High-volume forced diuresis with matched hydration using the RenalGuard system to prevent contrastinduced nephropathy: a meta-analysis of randomized trials," Clinical Cardiology, vol. 40, no. 12, pp. 1242-1246, 2017.

[17] S. S. Brar, V. Aharonian, P. Mansukhani et al., "Haemodynamic-guided fluid administration for the prevention of contrast-induced acute kidney injury: the POSEIDON randomised controlled trial," The Lancet, vol. 383, no. 9931, pp. 1814-1823, 2014.

[18] M. Maioli, A. Toso, M. Leoncini et al., "Bioimpedance-guided hydration for the prevention of contrast-induced kidney injury: the HYDRA study," Journal of the American College of Cardiology, vol. 71, no. 25, pp. 2880-2889, 2018.

[19] G. Qian, Z. Fu, J. Guo, F. Cao, and Y. Chen, "Prevention of contrast-induced nephropathy by central venous pressureguided fluid administration in chronic kidney disease and congestive heart failure patients," JACC: Cardiovascular Interventions, vol. 9, no. 1, pp. 89-96, 2016.

[20] J. Higgins and S. Green, Cochrane Handbook for Systematic Reviews of Interventions, The Cochrane Collaboration, London, UK, 2011.

[21] A. R. Jadad, R. A. Moore, D. Carroll et al., "Assessing the quality of reports of randomized clinical trials: is blinding necessary?," Controlled Clinical Trials, vol. 17, no. 1, pp. 1-12, 1996.

[22] A. E. Ades, M. Sculpher, A. Sutton et al., "Bayesian methods for evidence synthesis in cost-effectiveness analysis," PharmacoEconomics, vol. 24, no. 1, pp. 1-19, 2006.

[23] S. Brown, B. Hutton, T. Clifford et al., "A microsoft-excelbased tool for running and critically appraising network meta-analyses--an overview and application of NetMetaXL," Systematic Reviews, vol. 3, p. 110, 2014.

[24] M. S. van Mourik, F. van Kesteren, R. N. Planken et al., "Short versus conventional hydration for prevention of kidney injury during pre-TAVI computed tomography angiography," Netherlands Heart Journal, vol. 26, no. 9, pp. 425-432, 2018.

[25] A. Saratzis, V. Chiocchia, A. Jiffry et al., "HYDration and bicarbonate to prevent acute renal injury after endovascular aneurysm repair with suprarenal fixation: pilot/feasibility randomised controlled study (HYDRA pilot trial)," European Journal of Vascular and Endovascular Surgery, vol. 55, no. 5, pp. 648-656, 2018.

[26] J. Kooiman, J. P. M. de Vries, J. Van der Heyden et al., "Randomized trial of one-hour sodium bicarbonate vs standard periprocedural saline hydration in chronic kidney disease patients undergoing cardiovascular contrast procedures," PLoS One, vol. 13, Article ID e0189372, 2018.

[27] P. Alonso, J. Sanz, A. García-Orts et al., "Usefulness of sodium bicarbonate for the prevention of contrast-induced nephropathy in patients undergoing cardiac resynchronization therapy," The American Journal of Cardiology, vol. 120, no. 9, pp. 1584-1588, 2017.

[28] T. Usmiani, A. Andreis, C. Budano et al., "AKIGUARD (acute kidney injury GUARding device) trial: in-hospital and oneyear outcomes," Journal of Cardiovascular Medicine, vol. 17, no. 7, pp. 530-537, 2016.

[29] S. Turedi, E. Erdem, Y. Karaca et al., "The high risk of contrastinduced nephropathy in patients with suspected pulmonary embolism despite three different prophylaxis: a randomized controlled trial," Academic Emergency Medicine, vol. 23, no. 10, pp. 1136-1145, 2016.
[30] R. Solomon, P. Gordon, S. V. Manoukian et al., "Randomized trial of bicarbonate or saline study for the prevention of contrast-induced nephropathy in patients with CKD," Clinical Journal of the American Society of Nephrology, vol. 10, no. 9, pp. 1519-1524, 2015.

[31] P. L. Martin-Moreno, N. Varo, E. Martínez-Ansó et al., "Comparison of intravenous and oral hydration in the prevention of contrast-induced acute kidney injury in low-risk patients: a randomized trial," Nephron, vol. 131, no. 1, pp. 51-58, 2015.

[32] A. Jurado-Román, F. Hernández-Hernández, J. García-Tejada et al., "Role of hydration in contrast-induced nephropathy in patients who underwent primary percutaneous coronary intervention," The American Journal of Cardiology, vol. 115, no. 9, pp. 1174-1178, 2015.

[33] M. R. Yeganehkhah, L. Iranirad, F. Dorri et al., "Comparison between three supportive treatments for prevention of contrast-induced nephropathy in high-risk patients undergoing coronary angiography," Saudi Journal of Kidney Diseases and Transplantation: An Official Publication of the Saudi Center for Organ Transplantation, Saudi Arabia, vol. 25, no. 25, pp. 1217-1223, 2014.

[34] K. Yang, W. Liu, W. Ren, and S. Lv, "Different interventions in preventing contrast-induced nephropathy after percutaneous coronary intervention," International Urology and Nephrology, vol. 46, no. 9, pp. 1801-1807, 2014.

[35] P. Thayssen, J. F. Lassen, S. E. Jensen et al., "Prevention of contrast-induced nephropathy with $\mathrm{N}$-acetylcysteine or sodium bicarbonate in patients with ST-segment-myocardial infarction: a prospective, randomized, open-labeled trial," Circulation: Cardiovascular Interventions, vol. 7, no. 2, pp. 216-224, 2014.

[36] J. F. Nieto-Rios, W. A. Salazar, O. M. Sanchez et al., "Prevention of contrast induced nephropathy with sodium bicarbonate (the PROMEC study)," Jornal Brasileiro de Nefrologia, vol. 36, pp. 360-366, 2014.

[37] A. Manari, P. Magnavacchi, E. Puggioni et al., "Acute kidney injury after primary angioplasty: effect of different hydration treatments," Journal of Cardiovascular Medicine, vol. 15, no. 1, pp. 60-67, 2014.

[38] K. Mahmoodi, B. Sohrabi, F. Ilkhchooyi, M. Malaki, M. E. Khaniani, and M. Hemmati, "The efficacy of hydration with normal saline versus hydration with sodium bicarbonate in the prevention of contrast-induced nephropathy," Heart Views: The Official Journal of the Gulf Heart Association, vol. 15, no. 15, pp. 33-36, 2014.

[39] Y. Luo, X. Wang, Z. Ye et al., "Remedial hydration reduces the incidence of contrast-induced nephropathy and short-term adverse events in patients with ST-segment elevation myocardial infarction: a single-center, randomized trial," Internal Medicine, vol. 53, no. 20, pp. 2265-2272, 2014.

[40] J. Kooiman, Y. W. J. Sijpkens, J.-P. P. M. de Vries et al., “A randomized comparison of 1-h sodium bicarbonate hydration versus standard peri-procedural saline hydration in patients with chronic kidney disease undergoing intravenous contrastenhanced computerized tomography," Nephrology Dialysis Transplantation, vol. 29, no. 5, pp. 1029-1036, 2014.

[41] J. Kooiman, Y. W. J. Sijpkens, M. van Buren et al., "Randomised trial of no hydration vs. sodium bicarbonate hydration in patients with chronic kidney disease undergoing acute computed tomography-pulmonary angiography," Journal of Thrombosis and Haemostasis, vol. 12, no. 10, pp. 1658-1666, 2014. 
[42] S. Akyuz, T. Kemaloglu Oz, S. Altay et al., "Efficacy of oral hydration in the prevention of contrast-induced acute kidney injury in patients undergoing coronary angiography or intervention," Nephron Clinical Practice, vol. 128, no. 1-2, pp. 95-102, 2014.

[43] J. L. Kristeller, G. S. Zavorsky, J. E. Prior et al., "Lack of effectiveness of sodium bicarbonate in preventing kidney injury in patients undergoing cardiac surgery: a randomized controlled trial," Pharmacotherapy: The Journal of Human Pharmacology and Drug Therapy, vol. 33, no. 7, pp. 710-717, 2013.

[44] F. Koc, K. Ozdemir, F. Altunkas et al., "Sodium bicarbonate versus isotonic saline for the prevention of contrast-induced nephropathy in patients with diabetes mellitus undergoing coronary angiography and/or intervention: a multicenter prospective randomized study," Journal of Investigative Medicine, vol. 61, no. 5, pp. 872-877, 2013.

[45] G.-Q. Gu, R. Lu, W. Cui et al., "Low-dose furosemide administered with adequate hydration reduces contrast-induced nephropathy in patients undergoing coronary angiography," Cardiology, vol. 125, no. 2, pp. 69-73, 2013.

[46] P. Boucek, T. Havrdova, O. Oliyarnyk, J. Skibova, V. Pecenkova, and D. Sarkady, "Prevention of contrast-induced nephropathy in diabetic patients with renal function impairment: sodium bicarbonate versus sodium chloridebased hydration," Diabetologia, vol. 55, pp. S469-S70, 2012.

[47] G. Marenzi, C. Ferrari, I. Marana et al., "Prevention of contrast nephropathy by furosemide with matched hydration: the MYTHOS (induced diuresis with matched hydration compared to standard hydration for contrast induced nephropathy prevention) trial," JACC: Cardiovascular Interventions, vol. 5, no. 1, pp. 90-97, 2012.

[48] D.-G. Kong, Y.-F. Hou, L.-L. Ma, D.-K. Yao, and L.-X. Wang, "Comparison of oral and intravenous hydration strategies for the prevention of contrast-induced nephropathy in patients undergoing coronary angiography or angioplasty: a randomized clinical trial," Acta Cardiologica, vol. 67, no. 5, pp. 565-569, 2012.

[49] T. Klima, A. Christ, I. Marana et al., "Sodium chloride vs. sodium bicarbonate for the prevention of contrast mediuminduced nephropathy: a randomized controlled trial," European Heart Journal, vol. 33, no. 16, pp. 2071-2079, 2012.

[50] V. O. Gomes, R. Lasevitch, V. C. Lima et al., "Hydration with sodium bicarbonate does not prevent contrast nephropathy: a multicenter clinical trial," Arquivos Brasileiros de Cardiologia, vol. 99, no. 6, pp. 1129-1134, 2012.

[51] M. Motohiro, H. Kamihata, S. Tsujimoto et al., "A new protocol using sodium bicarbonate for the prevention of contrast-induced nephropathy in patients undergoing coronary angiography," The American Journal of Cardiology, vol. 107, no. 11, pp. 1604-1608, 2011.

[52] M. Maioli, A. Toso, M. Leoncini, C. Micheletti, and F. Bellandi, "Effects of hydration in contrast-induced acute kidney injury after primary angioplasty: a randomized, controlled trial," Circulation: Cardiovascular Interventions, vol. 4, no. 5, pp. 456-462, 2011.

[53] S.-W. Lee, W.-J. Kim, Y.-H. Kim et al., "Preventive strategies of renal insufficiency in patients with diabetes undergoing intervention or arteriography (the PREVENT Trial)," The American Journal of Cardiology, vol. 107, no. 10, pp. 14471452, 2011.

[54] A. M. Hafiz, M. F. Jan, N. Mori et al., "Prevention of contrastinduced acute kidney injury in patients with stable chronic renal disease undergoing elective percutaneous coronary and peripheral interventions: randomized comparison of two preventive strategies," Catheterization and Cardiovascular Interventions, vol. 79, no. 6, pp. 929-937, 2012.

[55] C. Briguori, G. Visconti, A. Focaccio et al., "Renal insufficiency after contrast media administration trial II (REMEDIAL II): RenalGuard system in high-risk patients for contrast-induced acute kidney injury," Circulation, vol. 124, no. 11, pp. 1260-1269, 2011.

[56] W. Wróbel, W. Sinkiewicz, M. Gordon, and A. WoniakWiniewska, "Oral versus intravenous hydration and renal function in diabetic patients undergoing percutaneous coronary interventions," Kardiologia Polska, vol. 68, pp. 10151020, 2010.

[57] A. Vasheghani-Farahani, G. Sadigh, S. E. Kassaian et al., "Sodium bicarbonate in preventing contrast nephropathy in patients at risk for volume overload: a randomized controlled trial," Journal of Nephrology, vol. 23, pp. 216-223, 2010.

[58] R. Cho, N. Javed, D. Traub, S. Kodali, F. Atem, and V. Srinivasan, "Oral hydration and alkalinization is noninferior to intravenous therapy for prevention of contrast-induced nephropathy in patients with chronic kidney disease," Journal of Interventional Cardiology, vol. 23, no. 5, pp. 460-466, 2010.

[59] A. Vasheghani-Farahani, G. Sadigh, S. E. Kassaian et al., "Sodium bicarbonate plus isotonic saline versus saline for prevention of contrast-induced nephropathy in patients undergoing coronary angiography: a randomized controlled trial," American Journal of Kidney Diseases, vol. 54, no. 4, pp. 610-618, 2009.

[60] A. Tamura, Y. Goto, K. Miyamoto et al., "Efficacy of singlebolus administration of sodium bicarbonate to prevent contrast-induced nephropathy in patients with mild renal insufficiency undergoing an elective coronary procedure," The American Journal of Cardiology, vol. 104, no. 7, pp. 921-925, 2009.

[61] M. Pakfetrat, M. H. Nikoo, L. Malekmakan et al., "A comparison of sodium bicarbonate infusion versus normal saline infusion and its combination with oral acetazolamide for prevention of contrast-induced nephropathy: a randomized, double-blind trial," International Urology and Nephrology, vol. 41, no. 3, pp. 629-634, 2009.

[62] M. Haase, A. Haase-Fielitz, R. Bellomo et al., "Sodium bicarbonate to prevent increases in serum creatinine after cardiac surgery: a pilot double-blind, randomized controlled trial," Critical Care Medicine, vol. 37, no. 1, pp. 39-47, 2009.

[63] P. Budhiraja, Z. Chen, and M. Popovtzer, "Sodium bicarbonate versus normal saline for protection against contrast nephropathy," Renal Failure, vol. 31, no. 2, pp. 118-123, 2009.

[64] D. Angoulvant, M. Cucherat, G. Rioufol et al., "Preventing acute decrease in renal function induced by coronary angiography (PRECORD): a prospective randomized trial," Archives of Cardiovascular Diseases, vol. 102, no. 11, pp. 761-767, 2009.

[65] M. Maioli, A. Toso, M. Leoncini et al., "Sodium bicarbonate versus saline for the prevention of contrast-induced nephropathy in patients with renal dysfunction undergoing coronary angiography or intervention," Journal of the American College of Cardiology, vol. 52, no. 8, pp. 599-604, 2008.

[66] S. L. Chen, J. Zhang, F. Yei et al., "Clinical outcomes of contrast-induced nephropathy in patients undergoing percutaneous coronary intervention: a prospective, multicenter, randomized study to analyze the effect of hydration and acetylcysteine," International Journal of Cardiology, vol. 126, no. 3, pp. 407-413, 2008. 
[67] S. S. Brar, A. Y. Shen, M. B. Jorgensen et al., "Sodium bicarbonate vs sodium chloride for the prevention of contrast medium-induced nephropathy in patients undergoing coronary angiography: a randomized trial," JAMA, vol. 300, no. 9, pp. 1038-1046, 2008.

[68] E. Adolph, B. Holdt-Lehmann, T Chatterjee et al., "Renal Insufficiency Following Radiocontrast Exposure Trial (REINFORCE): a randomized comparison of sodium bicarbonate versus sodium chloride hydration for the prevention of contrast-induced nephropathy," Coronary Artery Disease, vol. 19, no. 19, pp. 413-419, 2008.

[69] P. Schmidt, D. Pang, D. Nykamp, G. Knowlton, and H. Jia, "N-acetylcysteine and sodium bicarbonate versus $\mathrm{N}$-acetylcysteine and standard hydration for the prevention of radiocontrast-induced nephropathy following coronary angiography," Annals of Pharmacotherapy, vol. 41, no. 1, pp. $46-50,2007$.

[70] E. E. Ozcan, S. Guneri, B. Akdeniz et al., "Sodium bicarbonate, $\mathrm{N}$-acetylcysteine, and saline for prevention of radiocontrastinduced nephropathy. A comparison of 3 regimens for protecting contrast-induced nephropathy in patients undergoing coronary procedures. A single-center prospective controlled trial," American Heart Journal, vol. 154, no. 3, pp. 539-544, 2007.

[71] M. Masuda, T. Yamada, T. Mine et al., "Comparison of usefulness of sodium bicarbonate versus sodium chloride to prevent contrast-induced nephropathy in patients undergoing an emergent coronary procedure," The American Journal of Cardiology, vol. 100, no. 5, pp. 781-786, 2007.

[72] B. Dussol, S. Morange, A. Loundoun, P. Auquier, and Y. Berland, "A randomized trial of saline hydration to prevent contrast nephropathy in chronic renal failure patients," $\mathrm{Ne}$ phrology Dialysis Transplantation, vol. 21, no. 8, pp. 21202126, 2006.

[73] C. Mueller, P. Seidensticker, H. J Buettner et al., "Incidence of contrast nephropathy in patients receiving comprehensive intravenous and oral hydration," Swiss Medical Weekly, vol. 135, no. 135, pp. 286-290, 2005.

[74] G. J. Merten, W. P. Burgess, L. V. Gray et al., "Prevention of contrast-induced nephropathy with sodium bicarbonate: a randomized controlled trial," JAMA, vol. 291, no. 19, pp. 2328-2334, 2004.

[75] H. S. Trivedi, H. Moore, S. Nasr et al., "A randomized prospective trial to assess the role of saline hydration on the development of contrast nephrotoxicity," Nephron Clinical Practice, vol. 93, no. 93, pp. C29-C34, 2003.

[76] C. Mueller, G. Buerkle, H. J. Buettner et al., "Prevention of contrast media-associated nephropathy: randomized comparison of 2 hydration regimens in 1620 patients undergoing coronary angioplasty," Archives of Internal Medicine, vol. 162, no. 3, pp. 329-336, 2002.

[77] Task Force on Myocardial Revascularization of the European Society of C, the European Association for Cardio-Thoracic S, European Association for Percutaneous Cardiovascular I, "Guidelines on myocardial revascularization," European Journal of Cardio-Thoracic Surgery: Official Journal of the European Association for Cardio-Thoracic Surgery, vol. 38, pp. S1-S52, 2010.

[78] H. S. Thomsen, "European Society of Urogenital Radiology (ESUR) guidelines on the safe use of iodinated contrast media," European Journal of Radiology, vol. 60, no. 3, pp. 307-313, 2006.

[79] J. R. Brown, D. M. Pearlman, E. J. Marshall et al., "Metaanalysis of individual patient data of sodium bicarbonate and sodium chloride for all-cause mortality after coronary angiography," The American Journal of Cardiology, vol. 118, no. 10, pp. 1473-1479, 2016.

[80] B. Zhang, L. Liang, W. Chen, C. Liang, and S. Zhang, "The efficacy of sodium bicarbonate in preventing contrast-induced nephropathy in patients with pre-existing renal insufficiency: a meta-analysis," BMJ Open, vol. 5, Article ID e006989, 2015.

[81] S. Ali-Hassan-Sayegh, S. J. Mirhosseini, E. Rahimizadeh et al., "Current status of sodium bicarbonate in coronary angiography: an updated comprehensive meta-analysis and systematic review," Cardiology Research and Practice, vol. 2015, Article ID 690308, 16 pages, 2015.

[82] J.-S. Jang, H.-Y. Jin, J.-S. Seo et al., "Sodium bicarbonate therapy for the prevention of contrast-induced acute kidney injury-a systematic review and meta-analysis," Circulation Journal, vol. 76, no. 9, pp. 2255-2265, 2012.

[83] V. Kunadian, A. Zaman, I. Spyridopoulos, and W. Qiu, "Sodium bicarbonate for the prevention of contrast induced nephropathy: a meta-analysis of published clinical trials," European Journal of Radiology, vol. 79, no. 1, pp. 48-55, 2011.

[84] S. N. Heyman, S. Rosen, M. Khamaisi, J.-M. Idée, and C. Rosenberger, "Reactive oxygen species and the pathogenesis of radiocontrast-induced nephropathy," Investigative Radiology, vol. 45, no. 4, pp. 188-195, 2010. 